\title{
Different triggering mechanisms for solar flares and coronal mass ejections
}

\author{
E. Lippiello ${ }^{1}$, L. de Arcangelis ${ }^{1}$, and C. Godano ${ }^{2}$ \\ 1 Department of Information Engineering and CNISM, Second University of Naples, 81031 Aversa (CE), Italy \\ e-mail: dearcangelis@na.infn.it \\ 2 Department of Environmental Sciences and CNISM, Second University of Naples, 81100 Caserta, Italy \\ e-mail: cataldo.godano@unina2.it
}

Received 8 May 2008 / Accepted 15 July 2008

\section{ABSTRACT}

\begin{abstract}
Context. Experimental observations indicate that flares and CMEs are often associated, even if the precise relationship between the two phenomena is a still hot and open question.

Aims. We present a study of the statistical properties of solar flare and CME temporal organisation, in order to enlighten differences in the mechanisms at the basis of their occurrence.

Methods. We analyse experimental catalogs and compare the occurrence rate of flares and CME with the sunspot number. We also perform numerical simulations of simple stochastic models to be compared with experimental data.

Results. We find that the CME and flare occurrence rates are related, respectively, linearly and quadratically to the level of solar activity.

Conclusions. This behaviour could be understood in terms of correlations among flares: the occurrence of a flare, by perturbing the magnetic configuration, may eventually trigger further flares. This interpretation is supported by the existence of Omori sequences of after-flares following more energetic flares.
\end{abstract}

Key words. Sun: flares - Sun: coronal mass ejections (CMEs) - sunspots - methods: data analysis - methods: numerical methods: statistical

\section{Introduction}

The association between CMEs and flares (Kahler 1992; Harrison 2003; Harrison 2006) and their role for space weather (Gosling 1993; Pudovkin 1995) is an open and discussed problem. It is nowadays quite widely accepted that there exists a relation between CMEs and flares. However, most flares are not associated with CMEs and, at the same time, several CMEs are not flare related. The above findings leave the question of the cause-effect relationship between flares and CMEs still open and quite puzzling. Three scenarios have been proposed. In the flare-causing-CME scenario, the increment in temperature and density observed in a flare (Parker 1961) can produce a CME. In the CME-causing-flare scenario, conversely, flares are consequences of CME occurrence (Kahler 1992). In the third scenario (Harrison 1995) flares and CMEs are independent consequences of the same magnetic "disease". High quality experimental observations of solar corona (Zhang et al. 2001; Harrison et al. 2007) are compatible with the third, common cause scenario, but, if from one side they seem to reject the flare-causing-CME scenario, they do not definitely exclude the CME-causing-flare scenario. In the above standard approaches, the attempt is to discriminate among the possible scenarios by means of a precise localization in time and space of a CME and of eventually 'associated' flares. This is not always possible due to the absence of observations in the solar disk and low corona.

In this paper we address this problem with an alternative statistical approach, often adopted in the investigation of complex phenomena. Instead of focusing on a single event and, in particular, on the magnetic field evolution leading to a flare or a $\mathrm{CME}$, we analyse the temporal occurrence of a large statistical ensemble. Within this approach, the structure of the magnetic field is neglected but fundamental questions concerning, for instance, the existence of correlations among events are addressed. We analyse independently the statistical properties of flare and CME occurrence and their relation with the sunspot number, used as an index tracking the level of solar activity.

\section{Statistical analysis of experimental data}

Catalogs for flares, CMEs and sunspot numbers are available on line. In previous studies power law distributions of fundamental quantities have been observed (Aschwanden et al. 1998; Crosby et al. 1998; Lee et al. 1993; Boffetta et al. 1999; Wheatland 2003; Wheatland et al. 2002; de Arcangelis et al. 2006). In particular the energy distribution follows a power law for solar flares (Aschwanden et al. 1998), whereas it is consistent with a log-normal for CMEs (Gopalswamy 2006). In both cases, the functional form of the distribution does not appreciably change between the maximum and minimum phase. Moreover the association rate of CMEs and flares (Yashiro et al. 2005) and the frequency distribution of flares with and without associated CMEs (Yashiro et al. 2006) have been investigated. Here, we consider the sunspot number (http://www.ngdc.noaa.gov/) and flare data from GOES (Geostationary Operational Environmental Satellite) experiment (URL ftp: //ftp.ngdc.noaa.gov/STP/SOLAR_DATA/ SOLAR_FLARES), considering C1.4 class flares (peak flux larger 


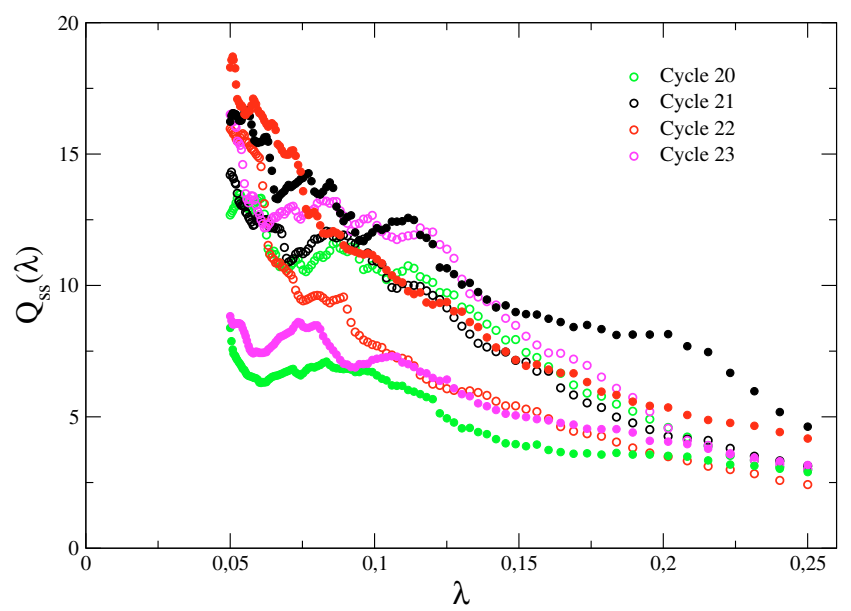

Fig. 1. (Color online) The rate-ratio $Q_{\mathrm{ss}}(\lambda)$ for sunspots as function of $\lambda$ for the ascending (empty circles) and the descending (filled circles) phases of cycles 20 to 23 . $Q_{\mathrm{ss}}(\lambda)$ exhibits similar behaviour for both phases of all cycles except for the descending phase of cycle 20 and 23.

then $1.4 \times 10^{-6} \mathrm{~W} \mathrm{~m}^{-2}$ ) in the solar cycles 21 (start in June 1976), 22 (start in September 1986) and 23 (start in May 1996). CMEs are available from the LASCO (Large Angle and Spectrometric Coronagraph) of the SOHO mission (URL http://cdaw. gsfc.nasa.gov/CME_list/) during solar cycle 23.

The occurrence rate, $R(t)$, for both CMEs and solar flares exhibits a periodic behaviour with the same period $T \simeq 11$ years of the sunspot number, with a minimum phase at the beginning of the solar cycle and a maximum phase centred at about $T / 2$. Comparison between the temporal behaviour can be obtained by plotting the rate of one phenomenon versus the corresponding rate of another in the same temporal window. Even applying a smoothing procedure, data turn out to be quite scattered. In order to reduce statistical fluctuations, we consider integrated quantities and define the rate-ratio $Q(\lambda)=R^{\max }(\lambda) / R^{\min }(\lambda)$, where for the ascending phase the integral $R^{\min }(\lambda)=\frac{1}{\lambda T} \int_{0}^{\lambda T} \mathrm{~d} t R(t)$ with $\lambda \leq 1 / 4$ is the average rate in the minimum. Analogously, the quantity $R^{\max }(\lambda)$ is the average rate over the interval $[T / 2-$ $\lambda T, T / 2]$ in the maximum. In a similar way we define the rate ratio in the descending phase, where $R^{\max }(\lambda)$ is now computed over the interval $[T / 2, T / 2+\lambda T]$ and $R^{\min }(\lambda)$ over $[T-\lambda T, T]$. The rate-ratio of two periodic phenomena with about the same time dependence will be simply proportional. Figure 1 shows the rate-ratio for sunspot numbers in different cycles plotted as function of $\lambda$. Curves for the ascending phase of all cycles show similar behaviour. In the small $\lambda$ regime, strong statistical oscillations are detected, which can be attributed to the narrow integration interval. Moreover, anomalous behaviour is found for the descending phases of cycles 20 and 23, where the sunspot rate ratio value is lower than expected. This result is consistent with other observations indicating a similar anomalous behaviour for the cycles 20 and 23 .

We then compute $Q(\lambda)$ for both solar flares and CMEs for various values of $\lambda \in[0.1,0.25]$. In Fig. 2 for each value of $\lambda$ we plot $Q_{\text {flares }}(\lambda)$ and $Q_{\mathrm{CME}}(\lambda)$ versus the rate-ratio for sunspot number, $Q_{\mathrm{ss}}(\lambda)$. It has been already observed for cycle 21 that the occurrence rate of CMEs is roughly proportional to sunspot number (Webb et al. 1994). This result is also observed for the ascending phase of cycle 23 . Indeed, Fig. 2 b shows that the rateratio for CMEs varies linearly with $Q_{\mathrm{ss}}(\lambda)$, i.e. a power law fit gives $Q_{\mathrm{CME}}(\lambda) \propto Q_{\mathrm{ss}}(\lambda)^{0.8}$. Conversely, a flat behaviour is detected for CME in the descending phase of cycle 23 . This could

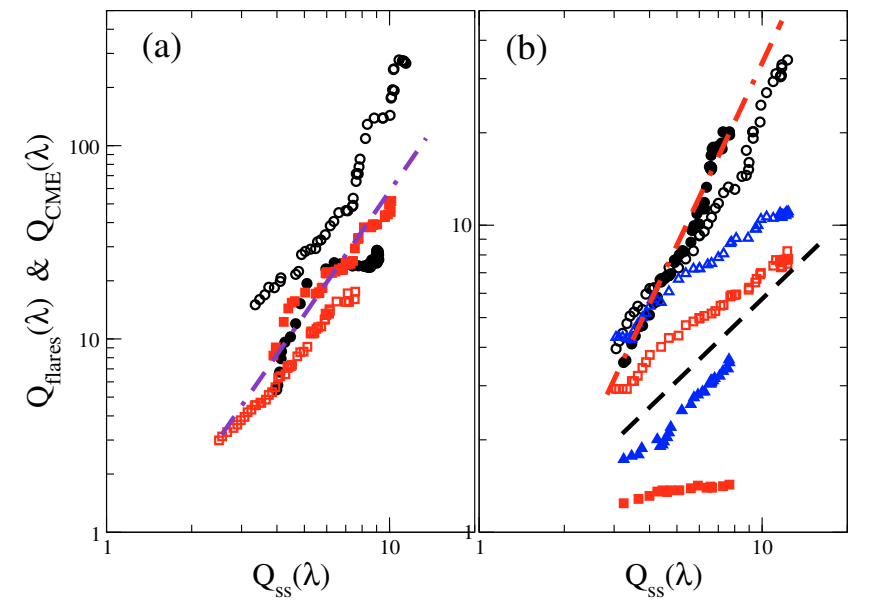

Fig. 2. (Color online) Panel a): $\log -\log$ plot of the rate-ratio $Q(\lambda)$ for class C1.4 flares of cycles 21 (black circles) and 22 (red squares) vs. rate-ratio of sunspots for different time intervals $\lambda T$. Empty and filled symbols refer to the ascending and descending phase respectively. Panel b): $\log -\log$ plot of the rate-ratio $Q(\lambda)$ for class C1.4 flares (black circles) and CMEs (red squares for data with no mass threshold, blue triangles for data with mass threshold $10^{10} \mathrm{~kg}$ ) of cycle $23 \mathrm{vs}$. rate-ratio of sunspots for different time intervals $\lambda T$. Empty and filled symbols refer to the ascending and descending phase respectively. $Q_{\mathrm{CME}}(\lambda)$ scales proportionally to $Q_{\mathrm{ss}}(\lambda)$ (dashed line has slope equal to 1 ). Rate-ratio for solar flares scales as the square of sunspot rate ratio (dot-dashed lines have slope equal to 2) for all solar cycles. $\lambda$ varies from 0.125 to 0.2 .

be attributed to the missing mass evaluation for all events occurred after June 2006. In order to overcome this problem, we have performed the same analysis only for CMEs with a measured mass larger than $10^{10} \mathrm{~kg}$. For the ascending phase, the introduction of a lower mass cutoff does not modify previous results. However, for the descending phase linear behaviour with the sunspot number is now recovered. As sunspots are a tracer of the level of magnetic activity, the result suggests that CME occurrence linearly follows the temporal evolution of Sun's magnetic energy.

A strikingly different behaviour is observed for flares. For all analysed cycles in both the ascending and the descending phases, $Q_{\text {flares }}(\lambda)$ exhibits an enhanced relationship with the sunspot rate ratio. More precisely, data are always consistent with a power law behaviour $Q_{\text {flares }}(\lambda) \sim Q_{\text {ss }}(\lambda)^{\alpha}$ with $\alpha \in[1.7,2.3]$ and on average $\alpha \simeq 2$.

A possible explanation for the flare-sunspot enhanced relationship is the presence of correlations between flares. This is in agreement with the recent finding that the rate of occurrence of low energy flares increases when a large flare occurs and then decays in time as a power law (de Arcangelis et al. 2006). This is the analogue of the Omori law for seismic occurrence and suggests that after a large (main) flare the occurrence probability of other events becomes higher, leading to a sequence of after-flares following a main-flare. We here extend the analysis performed for flares to CMEs. We define a "main" flare an event with energy larger than a given threshold $e_{\mathrm{th}}$ and similarly a "main" CME an event with mass larger than $m_{\mathrm{th}}$. For given $e_{\mathrm{th}}$ and $m_{\mathrm{th}}$, one identifies a set of $L$ main events each occurring at time $t_{i}$. We then compute the quantity

$\mathcal{N}\left(t, e_{\mathrm{th}}\right)=\sum_{i=1}^{L} n\left(t-t_{i}\right) \Theta\left(t-t_{i+1}\right)$ 


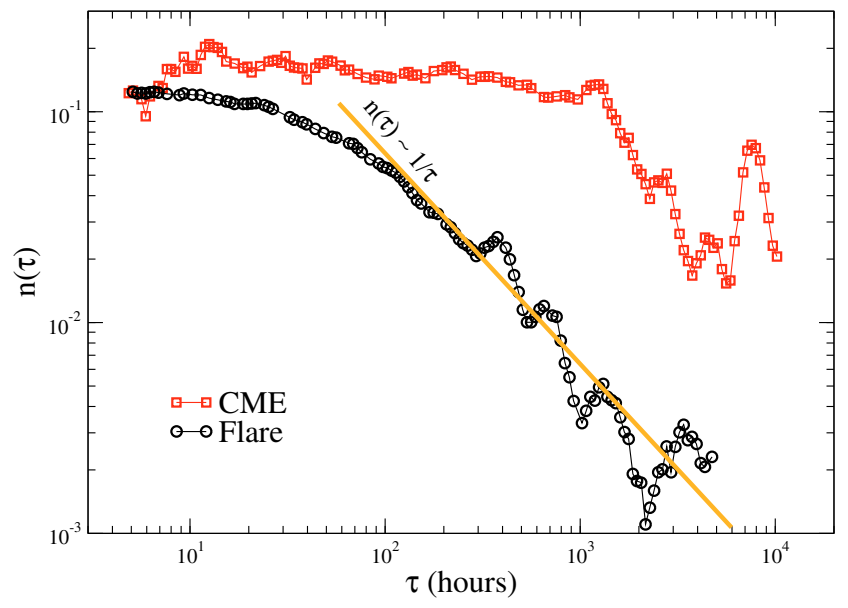

Fig. 3. (Color online) The $\log -\log$ plot of the after-flare distribution (black circles) defined in Eq. (5) for class $\mathrm{M}$ main flares $\left(e_{\mathrm{th}} \geq\right.$ $10^{-5} \mathrm{~W} / \mathrm{m}^{2}$ ). The power law $n(\tau) \sim \tau^{-1}$ (orange straight line), reminiscent of the Omori law for seismic sequences, fits experimental data for $\tau>10^{2}$ h. $n(\tau)$ similarly defined for CME with $m_{\mathrm{th}}=10^{13} \mathrm{~kg}$ exhibits an about flat behaviour very different from the one observed in flare occurrence.

where $\Theta(x)$ is the Heaviside step function and $n\left(t-t_{i}\right)$ is the number of flares with energy smaller than $e_{\text {th }}$ occurring at time $t-t_{i}$ after the $i$ th main flare. Assuming that $n\left(t-t_{i}\right)$ is time translational invariant, from Eq. (1) one obtains

$n(\tau)=\frac{\mathcal{N}\left(t, e_{\mathrm{th}}\right)}{\sum_{i=1}^{L} \Theta\left(t-t_{i+1}\right)}$

where $\tau$ is the time elapsed from the main event. An analogous definition is used for CME occurrence with the flare energy threshold $\left(e_{\mathrm{th}}\right)$ replaced by the CME mass threshold $\left(m_{\mathrm{th}}\right)$. The results for $n(\tau)$ with $e_{\mathrm{th}}=10^{-5} \mathrm{~W} / \mathrm{m}^{2}$ (class M-flares) and for $m_{\text {th }}=10^{13} \mathrm{~kg}$ are plotted in Fig. 3. This figure clearly shows differences in the behaviour of the two phenomena. In the case of flares, $n(\tau)$ exhibits a power law decay $n(\tau) \sim 1 / \tau$ over almost two orders of magnitude, whereas $n(\tau)$ for CMEs has a substantially flat behaviour, except in the last decade where statistical fluctuations are large. Both results are quite robust with respect to different choices for $e_{\text {th }}$ and $m_{\text {th }}$. The power law behaviour $n(\tau) \propto 1 /(\tau+c)$, that very well fits flare data, is a typical feature of seismic occurrence, known as the Omori law. Commonly, in seismicity the delay time $c$ is attributed to undetected events soon after the mainshock. Similarly, one could interpret the flat behaviour observed at short times in Fig. 3 as the effect of obscuration of small flares in the decay phase of large flares (Wheatland 2001). The Omori decay is generally interpreted in seismicity in terms of energy redistribution mechanisms: earthquakes can be viewed as a relaxation process of the earth crust loaded by the tectonic motion at lithospheric plate boundaries. When an earthquake occurs, the stress in the crust is redistributed generating new earthquakes where and when the stress assume again a value greater than the slipping threshold. The similar power law decays of $n(\tau)$ for flares, suggests an analogy between earthquake and flare occurrence, where the drive due to the magnetic energy plays the role of tectonic motion and magnetic energy the role of mechanic stress redistribution. This redistribution should be responsible of the enhanced relationship flare-sunspot rate whereas it seems not relevant in CME occurrence.

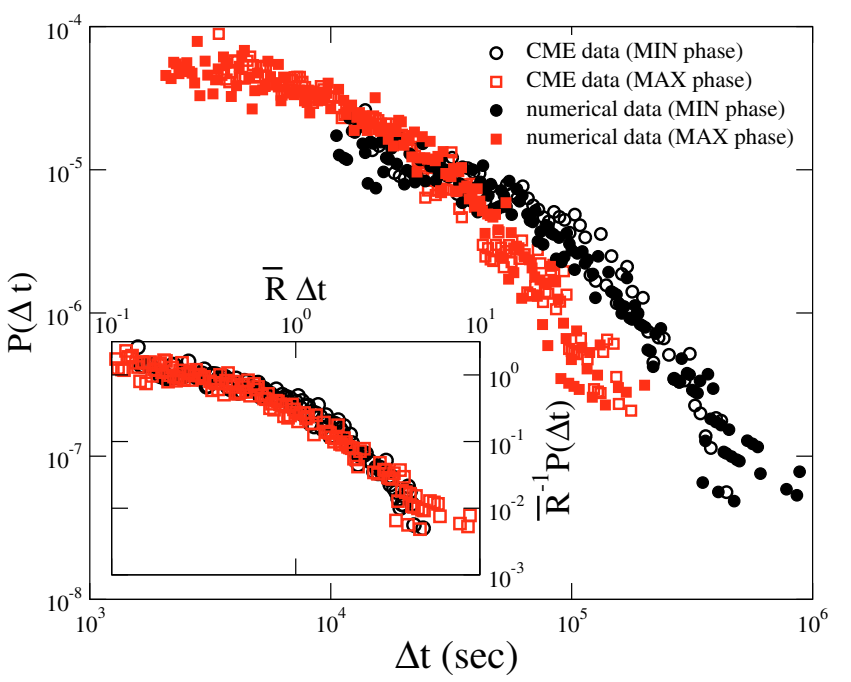

Fig. 4. (Color online) Distributions $P(\Delta t)$ of waiting times $\Delta t$ between two successive CMEs, for the maximum and minimum phase. Data for the numerical catalog generated according to the probability Eq. (1), proportional to the sunspot number, are plotted with filled symbols. Numerical data exhibit good collapse with experimental CME data in both phases. In the inset, distributions normalized by the average rate $\bar{R}$, vs. $\bar{R} \Delta t$ are plotted according to Eq. (2). Rescaled distributions for the maximum and minimum phase show very good collapse.

\section{Theoretical model and rescaling}

To support the above interpretation, we will show that CME occurrence can be described by a piece-wise Poissonian process (Wheatland et al. 2002) where non-interacting events occur with a probability

$p_{\mathrm{CME}}(t)=K n_{\mathrm{ss}}(t)$

where $n_{\mathrm{ss}}(t)$ is the monthly average of sunspot number and $K$ is an a-dimensional constant. The same approach fails in the description of flare occurrence.

In order to test the above scenario, we generate via numerical simulations a catalog where events randomly occur according to the probability in Eq. (3). We notice that the only free parameter is the constant $K$, whose value is fixed imposing that the numerical catalog contains the same total number of events as the CME experimental one. We compare the temporal organisation of the experimental and numerical catalogs by analysing the distribution of waiting times $P(\Delta t)$, where $\Delta t$ is defined as the time elapsed between two successive events. This quantity is often introduced to characterize the temporal occurrence of stochastic processes and it has been extensively investigated in the context of solar physics (Wheatland 2003, 2002; Boffetta 1999; Lepreti 2001; de Arcangelis 2006; Baiesi 2006). More precisely, $P(\Delta t)$ has been evaluated for both flares and CMEs in the maximum and minimum phase. The functional form is essentially similar for the two phenomena, with the tail of the distribution fitted by power laws (Wheatland 2003; Wheatland et al. 2002; Baiesi et al. 2006). Furthermore, the distribution of intertimes between energetic flares occurring in individual active regions can be approximated by an exponential distribution (Moon et al. 2001). We separately compute the $P(\Delta t)$ inside the time windows [0, $T / 4]$ (minimum phase) and [T/4, $T / 2]$ (maximum phase). Analogous results are obtained for the descending phase. Data are shown for both the experimental CME and the numerically generated catalog (Fig. 4). Excellent agreement is found between numerical and experimental data in both phases. 


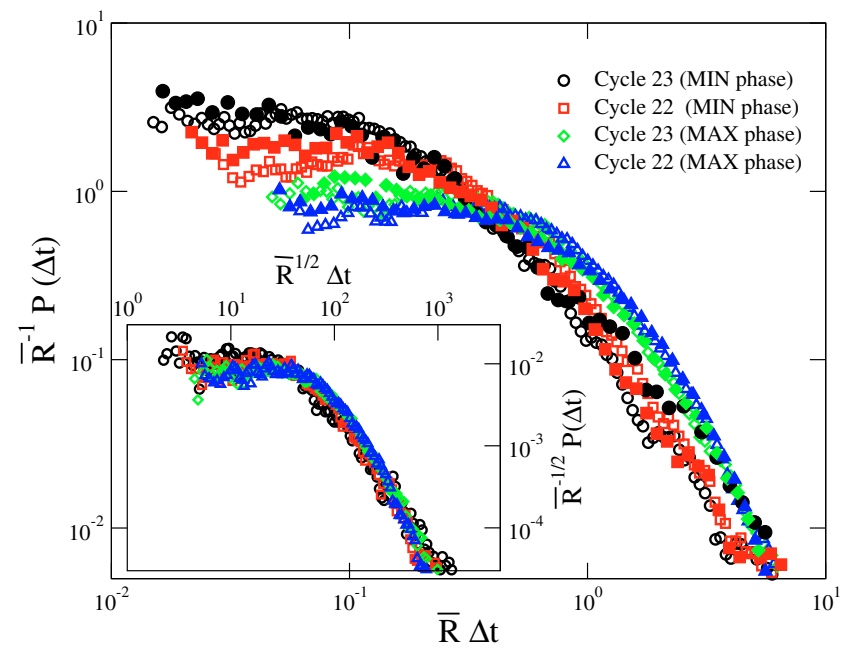

Fig. 5. (Color online) Distribution $P(\Delta t)$ of waiting times $\Delta t$ between two successive class C1.4 flares, normalized by the average rate $\bar{R}$, vs. $\bar{R} \Delta t$ plotted according to Eq. (2). Data for the maximum and minimum phase for both cycle 22 and cycle 23 do not collapse onto a unique curve. Numerical data (filled symbols), obtained according to the probability Eq. (3), reproduce experimental results in both solar cycles and solar phases. Inset: good collapse is obtained if data are rescaled with the square root of the average rate $\bar{R}^{1 / 2}$.

Hence, without any parameter tuning, a generalized Poissonian process with a rate proportional to the Sun's magnetic energy reproduces $\mathrm{CME}$ temporal occurrence. In the inset we show the waiting time distribution rescaled by $\bar{R}$, the average rate inside each phase. The inverse average rate represents a characteristic time for the stochastic process. The collapse between minimum and maximum phase data indicates that $\mathrm{CME}$ occurrence verifies the property

$P(\Delta t)=\bar{R} f(\bar{R} \Delta t)$.

This property is straightforward for a homogeneous Poisson process with an exponential $f(x)$. However it has been observed in other complex non Poissonian phenomena, where the scaling function $f(x)$ shows non-exponential universal behaviour (Corral 2004).

The above analysis performed for solar flares provides a strikingly different result. Figure 5 indeed shows that Eq. (4) is no longer fulfilled by solar flares, i.e. data corresponding to different phases do not collapse (Baiesi et al. 2006). As a consequence, since the stochastic process with a rate given by Eq. (3) obeys the scaling relation Eq. (4), this process fails to describe flare occurrence. On the other hand, the non linear relationship between flare and sunspot rate (Fig. 2) suggests a stochastic process different from Eq. (3) to characterize flaring occurrence

$p_{\text {flare }}(t) \simeq K_{2}\left(n_{\mathrm{ss}}(t)\right)^{2}$.

We generate a numerical catalog with the probability given by the above equation and compare it with experimental data for flares. Figure 5 shows indeed good agreement between the experimental and numerical waiting time distribution in both solar phases and solar cycles considered. According to this finding one then expects that the flare rate approximately grows as $R_{\text {flares }} \sim R_{\mathrm{CME}}^{2}$ and therefore $\bar{R}^{1 / 2}$ for flares should correspond to the characteristic time for CME occurrence. Therefore, since
CMEs follow Eq. (4), this scaling should be recovered also for flares if one replaces $\bar{R}$ by its square root. Performing this substitution, a good collapse is indeed obtained not only between the two different solar phases but also for different solar cycles (inset of Fig. 5).

\section{Conclusions}

A statistical analysis of experimental catalogs and numerical simulations clearly indicate important differences in the temporal organisation of flares and CMEs. CME occurrence is indeed very well described by a piece-wise Poissonian process with a rate proportional to sunspots. Conversely, flares display non-trivial temporal correlations, giving rise to Omori-like behaviour after the occurrence of energetic flares. As a consequence of the above scenario, Sun's magnetic field that causes the reconnection of twisted magnetic loops, is able to account for observed CME occurrence. On the other hand, our statistical analysis suggests that the energy redistributed by a flare could enhance further reconnections, triggering successive correlated flares. Energy redistribution can be related to the modification of the field topology within the active region produced by the magnetic reconnection of a given flux tube. Details of magnetic topology at the basis of this mechanism are beyond this approach, which is however able to invalidate all models with identical triggering mechanism for both phenomena. For instance, avalanche-type models with a time dependent drive (Lu et al. 1991; Norman et al. 2001) can apply to CMEs but do not provide a complete characterization of flare correlations.

\section{References}

Aschwanden, M. J., Dennis, B. R., \& Benz, A. O. 1998, ApJ, 497, 972

Baiesi, M., Paczuski, M., \& Stella, A. 2006, Phys. Rev. Lett., 96, 0511031

Boffetta, G., Carbone, V., Giuliani, P., Veltri, P., \& Vulpiani, A. 1999, Phys. Rev. Lett., 83, 4662

Corral, A. 2004, Phys. Rev. Lett., 92, 1085011

Crosby, N., Vilmer, N., Lund, N., \& Sunyaev, R. 1998, A\&A, 334, 299

de Arcangelis, L., Godano, C., Lippiello, E., \& Nicodemi, M. 2006, Phys. Rev. Lett., 96, 0511021

Gopalswamy, N. 2006, J. A\&A, 27, 243

Gosling, J. T. 1993, J. Geophys. Res., 98, 937

Harrison, R. A. 1995, A\&A, 304, 585

Harrison, R. A. 2003, Adv. Space Res., 32, 2425

Harrison, R. A. 2006, in Solar Eruptions and Energetic Particles, ed. N. Gopalswamy, R. Mewaldt, \& J. Torsti (AGU Geophysical monograph), 165 Harrison, R. A., \& Bewsher, D. 2007, A\&A, 461, 1155

Kahler, S. W. 1992, ARA\&A, 30, 113

Lee, T. T., Petrosian, V., \& McTiernan, J. M. 1993, ApJ, 412, 401

Lepreti, F., Carbone, V., \& Veltri, P. 2001, ApJ, 555, L133

Lu, E. T., \& Hamilton, R. J. 1991, ApJ, 380, L89

Moon, Y. J., Choe, G. S., Yun, H. S., \& Park, Y. D. 2001, J. Geophys. Res., 106, 29951

Norman, J. P., Charbonneau, P., McIntosh, S. W., \& Liu, H. 2001, ApJ, 557, 891

Parker, E. N. 1961, ApJ, 133, 1014

Pudovkin, M. I. 1995, J. Geophys. Res., 100, 7917

Webb, D. F., \& Howard, R. A. 1994, J. Geophys. Res., 99, 4201

Wheatland, M. S. 2001, Sol. Phys., 203, 87

Wheatland, M. S. 2003, Sol. Phys., 214, 361

Wheatland, M. S., \& Litvinenko, Y. E. 2002, Sol. Phys., 211, 255

Yashiro, S., Gopalswamy, N., Akiyama, S., Michalek, G., \& Howard, R. A. 2005, J. Geophys. Res., 105, A12S051

Yashiro, S., Akiyama, S., Gopalswamy, N., \& Howard, R. A. 2006, ApJ, 650, L143

Zhang, J., Dere, K. P., Howard, R. A., Kundu, M. R., \& White, S. M. 2001, ApJ, 559,452 